\title{
Multivariable orthogonal polynomials associated with tensor products of the oscillator algebra $\mathfrak{b}(1)$
}

\author{
S. Lievens and J. Van der Jeugt \\ Department of Applied Mathematics and Computer Science, \\ University of Ghent, Krijgslaan 281-S9, B-9000 Gent, Belgium. \\ E-mails : Stijn.Lievens@ugent.be, Joris.VanderJeugt@ugent.be.
}

\begin{abstract}
It is well known that the tensor product of $n+1$ lowest weight representations of (a generalization of) the oscillator algebra $\mathfrak{b}(1)$ can be depicted by a binary coupling tree. With each such binary coupling tree, we associate a polynomial $R_{l}^{(h)}(x)$ that will turn out to be an $n$-variable Hermite polynomial. We prove that the polynomials $R_{l}^{(h)}(x)$ associated with a fixed binary coupling tree are orthogonal over the $n$-dimensional real space for some weight function, that is independent of the shape of the considered binary coupling tree. The connection coefficients expressing a polynomial associated with a given binary coupling in terms of polynomials associated with another binary coupling tree are the $3 n j$-coefficients of $\mathfrak{b}(1)$.
\end{abstract}

Running title: Multivariable orthogonal polynomials for $\mathfrak{b}(1)$

PACS: 02.20.+b, 02.30.+g, 03.65.Fd. 


\section{Introduction}

General recoupling coefficients or $3 n j$-coefficients of Lie algebras play an important role in theoretical physics, but also have many mathematical applications, e.g. the $3 j$ and $6 j$ coefficients of $\mathfrak{s u}(2)$ exhibit a close relationship with Hahn and Racah polynomials $[1,2]$. In [3] Granovskiı̌ and Zhedanov give a new method for the construction of $3 n j$-symbols. In [4] their method was applied to the Lie algebras $\mathfrak{s u}(1,1)$ and $\mathfrak{b}(1)$ to find addition formulas for various orthogonal polynomials. Generalizing the most important formulas in this paper, we showed in [5] that one can associate multivariable Jacobi and continuous Hahn polynomials with tensor products of positive discrete series representations of the Lie algebra $\mathfrak{s u}(1,1)$. A convenient way to describe the order of taking these tensor products (or couplings) is the use of binary coupling trees, see [6, Topic 12] and [7, 8]. We will use tree terminology (e.g. leaf nodes, root node, subtree,... ) that should be self-explanatory, but in doubt see [9].

In this article, we will follow the same technique as in [5] to find multivariable Hermite polynomials that are associated with tensor products of lowest weight representations of (an extension of) the oscillator algebra. We will show that these multivariable Hermite polynomials are orthogonal on $\mathbb{R}^{n}$ for a certain weight function that is independent of the order of taking tensor products, although the polynomials themselves are dependent on this order. The connection coefficients between the different classes of polynomials will turn out to be (just as in [5]) the $3 n j$-coefficients of the oscillator algebra.

In general, orthogonal polynomials in several variables give rise to certain difficulties that are not present in the one variable situation. For example, orthogonal polynomials in $n$ variables are no longer uniquely defined by the region $\Omega$ and the weight function on the region. This is closely related to the fact that there is no obvious natural order for polynomials in several variables.

The space of all polynomials in the variables $x_{1}, \ldots, x_{n}$ with real coefficients is denoted $\mathbb{R}\left[x_{1}, \ldots, x_{n}\right]$ or $\Pi^{n}$ for short. The degree of a polynomial $P \in \Pi^{n}$ is the highest degree of 
any of its monomials. Let $\langle\cdot, \cdot\rangle$ be an inner product defined on $\Pi^{n}$, then $P$ is an orthogonal polynomial if $\langle P, Q\rangle=0$ for all polynomials $Q$ with $\operatorname{deg} Q<\operatorname{deg} P$. This definition does not require that $P$ is orthogonal with other (orthogonal) polynomials of the same degree.

In our case the inner product will be defined in terms of some (classical) weight function $W$ on some (classical) region $\Omega$ in $\mathbb{R}^{n}:\langle P, Q\rangle=\int_{\Omega} P(x) Q(x) W(x) d x$.

The rest of this article is organized as follows: In Section II we give the definition of the Lie algebra $\mathfrak{b}(1)$ and a class of representations, together with expressions for its ClebschGordan and Racah coefficients. In Section III the method of Granovskil and Zhedanov is briefly explained and a (new) addition formula for Meixner polynomials is found. Whereas in Section III we confine ourselves to the tensor product of three representations, we turn in Section IV to the multivariable case. We explain how to associate a multivariable Hermite polynomial to a binary coupling tree and prove the orthogonality of these polynomials. In the last Section, we show how this leads to an integral formula for $3 n j$-coefficients of $\mathfrak{b}(1)$.

We conclude this introduction with some notational conventions. The notation for (generalized) hypergeometric functions is the standard one $[10,11]$ :

$$
{ }_{p} F_{q}\left(\begin{array}{l}
a_{1}, \ldots, a_{p} \\
b_{1}, \ldots, b_{q}
\end{array} ; z\right)=\sum_{k=0}^{\infty} \frac{\left(a_{1}\right)_{k} \cdots\left(a_{p}\right)_{k}}{\left(b_{1}\right)_{k} \cdots\left(b_{q}\right)_{k}} \frac{z^{k}}{k !},
$$

with $(a)_{k}$ the Pochhammer symbol: $(a)_{k}=a(a+1) \cdots(a+k-1)$ for $k>0$, and $(a)_{0}=1$. Convergence issues will not arise since all the hypergeometric functions in this article are terminating (i.e. one of the numerator parameters is a negative integer). Furthermore, for the one-dimensional orthogonal polynomials appearing in this article, we will adopt the notation and normalization of [12]. In particular, we denote the Hermite polynomials by $H_{n}(x)$, with:

$$
H_{n}(x)=(2 x)_{2}^{n} F_{0}\left(\begin{array}{c}
-n / 2,-(n-1) / 2 \\
-
\end{array}-\frac{1}{x^{2}}\right) .
$$

These are orthogonal on $\mathbb{R}$ for the weight function $\exp \left(-x^{2}\right)$ :

$$
\int_{-\infty}^{\infty} H_{n}(x) H_{m}(x) e^{-x^{2}} d x=\sqrt{\pi} 2^{n} n ! \delta_{n, m}
$$




\section{The Lie algebra $\mathfrak{b}(1)$ and tensor products for a class of representations}

The Lie algebra $\mathfrak{b}(1)[13,4]$ is four-dimensional, with basis elements $b^{ \pm}, H$ and $N$ subject to the relations

$$
\left[b^{-}, b^{+}\right]=H, \quad\left[N, b^{ \pm}\right]= \pm b^{ \pm} \text {and }[H, x]=0, \text { for } x=b^{ \pm}, N
$$

where the basis elements obey the following unitary conditions $H^{\dagger}=H, N^{\dagger}=N$ and $\left(b^{ \pm}\right)^{\dagger}=b^{\mp}$ (more precisely: the Lie algebra $\mathfrak{b}(1)$ is a $\star$-algebra with $\star$-operation defined by $H^{\star}=H, N^{\star}=N$ and $\left(b^{ \pm}\right)^{\star}=b^{\mp}$; then the representation operators corresponding to the Lie algebra elements satisfy the same conjugacy properties in the $\star$-representations considered here).

It is well known that this Lie algebra has a class of lowest weight representations [13], characterized by two positive numbers $h$ and $k$. These representations are infinite dimensional and have a set of orthonormal basis vectors $|h, k, n\rangle$, with $n=0,1,2, \ldots$ The action of the operators corresponding to the Lie algebra basis elements on these basis vectors is given by

$$
\left\{\begin{aligned}
H|h, k, n\rangle & =h|h, k, n\rangle \\
N|h, k, n\rangle & =(k+n)|h, k, n\rangle \\
b^{-}|h, k, n\rangle & =\sqrt{h n}|h, k, n-1\rangle \\
b^{+}|h, k, n\rangle & =\sqrt{h(n+1)}|h, k, n+1\rangle
\end{aligned}\right.
$$

One can verify that these representations are unitary and irreducible when $h>0$ and $k \geq 0$.

Taking the tensor product of two such unitary and irreducible representations $\left(h_{1}, k_{1}\right)$ and $\left(h_{2}, k_{2}\right)$ gives again a unitary representation that is completely reducible into representations of the same type:

$$
\left(h_{1}, k_{1}\right) \otimes\left(h_{2}, k_{2}\right)=\bigoplus_{j=0}^{\infty}\left(h_{1}+h_{2}, k_{1}+k_{2}+j\right) .
$$


We note that the $h$-labels are simply additive and that the difference between the resulting and consisting $k$-labels is a nonnegative integer.

Now, one can express a basis vector $\left|h_{1}+h_{2}, k_{1}+k_{2}+j, n\right\rangle$ in terms of tensor product vectors $\left|h_{1}, k_{1}, n_{1}\right\rangle \otimes\left|h_{2}, k_{2}, n_{2}\right\rangle$. The coefficients accomplishing this connection are called the Clebsch-Gordan coefficients. Explicitly one has, in an obvious notation with $k_{12}=$ $k_{1}+k_{2}+j$,

$\left|\left(h_{1}, h_{2}\right), k_{12}, n\right\rangle \equiv\left|\left(h_{1}, k_{1} ; h_{2}, k_{2}\right) h_{1}+h_{2}, k_{1}+k_{2}+j, n\right\rangle=\sum_{n_{1}, n_{2}} C_{n_{1}, n_{2}, n}^{h_{1}, h_{2}, j}\left|h_{1}, k_{1}, n_{1}\right\rangle \otimes\left|h_{2}, k_{2}, n_{2}\right\rangle$

where the sum is over all positive integers $n_{1}$ and $n_{2}$ such that $n_{1}+n_{2}=n+j$. The labels $k_{1}$ and $k_{2}$ have been omitted from the notation of the Clebsch-Gordan coefficients as they are independent of them $[4$, Section VI]. An explicit expression is given by:

$$
C_{n_{1}, n_{2}, n}^{h_{1}, h_{2}, j}=\delta_{n_{1}+n_{2}, j+n}(-1)^{n_{2}} \frac{\left(n_{1}+n_{2}\right) ! a^{n+n_{2}} b^{n_{1}-n}}{\sqrt{n_{1} ! n_{2} ! j ! n !}}{ }_{2} F_{1}\left(\begin{array}{c}
-n,-n_{2} \\
-j-n
\end{array} ; \frac{1}{a^{2}}\right),
$$

with

$$
a=\sqrt{\frac{h_{1}}{h_{1}+h_{2}}}, \quad b=\sqrt{\frac{h_{2}}{h_{1}+h_{2}}}, \quad \text { and thus } a^{2}+b^{2}=1 .
$$

We remark that although the denominator parameter in (2.8) is a negative integer, the hypergeometric notation is still well-defined because the denominator parameter is smaller than the numerator parameters, which determine the termination.

The tensor product of three representations $\left(h_{1}, k_{1}\right),\left(h_{2}, k_{2}\right)$ and $\left(h_{3}, k_{3}\right)$ can be formed in two different ways:

$$
\left(\left(h_{1}, k_{1}\right) \otimes\left(h_{2}, k_{2}\right)\right) \otimes\left(h_{3}, k_{3}\right), \text { and }\left(h_{1}, k_{1}\right) \otimes\left(\left(h_{2}, k_{2}\right) \otimes\left(h_{3}, k_{3}\right)\right) .
$$

The coefficients relating the two coupled bases, for which the notation is self-explanatory, are called the recoupling or Racah coefficients:

$$
\left|\left(\left(h_{1}, h_{2}\right) h_{3}\right), k_{12}, k, n\right\rangle=\sum_{k_{23}=k_{2}+k_{3}}^{k-k_{1}} h_{1}, h_{2}, h_{3} U_{k_{3}, k, k_{23}}^{k_{1}, k_{2}, k_{12}}\left|\left(h_{1}\left(h_{2}, h_{3}\right)\right), k_{23}, k, n\right\rangle .
$$


This representation of the Lie algebra $\mathfrak{b}(1)$ has the remarkable property that its Racah coefficients are of the same type as its Clebsch-Gordan coefficients [4, Section VII]. One has

$$
h_{1}, h_{2}, h_{3} U_{k 3, k, k_{23}}^{k_{1}, k_{2}, k_{12}}=(-1)^{j_{12}} \frac{\left(j+j_{12}\right) ! \tilde{a}^{j_{12}+j^{\prime}} \tilde{b}^{j-j^{\prime}}}{\sqrt{j_{12} ! j_{23} ! j ! j^{\prime} !}}{ }_{2} F_{1}\left(\begin{array}{c}
-j_{12},-j^{\prime} \\
-j_{12}-j
\end{array} ; \frac{1}{\tilde{a}^{2}}\right)
$$

with

$$
\tilde{a}=\sqrt{\frac{h_{1} h_{3}}{\left(h_{1}+h_{2}\right)\left(h_{2}+h_{3}\right)}}, \quad \tilde{b}=\sqrt{\frac{h_{2}\left(h_{1}+h_{2}+h_{3}\right)}{\left(h_{1}+h_{2}\right)\left(h_{2}+h_{3}\right)}}, \quad \text { and thus also } \tilde{a}^{2}+\tilde{b}^{2}=1 .
$$

Moreover, we denoted the nonnegative integer differences by

$$
j_{12}=k_{12}-k_{1}-k_{2}, j=k-k_{12}-k_{3}, j_{23}=k_{23}-k_{2}-k_{3} \text { and } j^{\prime}=k-k_{1}-k_{23},
$$

and thus $j_{12}+j=j_{23}+j^{\prime}$. We remark that the recoupling coefficients are only dependent on these integer differences (and not on the $k$-labels themselves). We choose however to adopt a notation for the recoupling coefficients of $\mathfrak{b}(1)$ that resembles that of the recoupling coefficients of $\mathfrak{s u}(1,1)$ in [5].

It is known [1] (and also immediately clear from the expressions given here) that both the Clebsch-Gordan and Racah coefficients of $\mathfrak{b}(1)$ can be written in terms of the Krawtchouk polynomials [12].

\section{Construction of convolution identities}

In the seminal paper [3] a new method for the construction of $3 n j$-symbols was introduced and applied to the $\mathfrak{s u}(1,1)$ case. This method can be extended to other Lie algebras, and in [14] it was used to derive a generating function for the $9 j$-symbols of the oscillator algebra. In $[4,15]$ this method was used to find addition formulas or convolution identities for orthogonal polynomials.

In short: starting with a certain operator $X$ acting on the representation space of the Lie algebra, one finds a quantity $Q_{n}(x)$ that is the remainder of the overlap coefficient between 
an eigenvector of $X$ and the basis vector $|n\rangle$ after factoring out the "zero-amplitude" part. E.g. in the $\mathfrak{b}(1)$ case

$$
\langle h, k, x \mid h, k, n\rangle=\langle h, k, x \mid h, k, 0\rangle Q_{n}(x ; h, k) .
$$

For certain choices of $X$, the quantity $Q$ will be a classical orthogonal polynomial. However, this need not be so, and interesting applications exist without $Q$ being an orthogonal polynomial [14]. Next, one uses this polynomial $Q$ and the (zero-amplitude) ClebschGordan coefficients of the considered Lie algebra to define a two-variable polynomial $S_{j}\left(x_{1}, x_{2}\right)$ that satisfies the important relation (written down for the $\mathfrak{b}(1)$ case)

$$
\begin{aligned}
& S_{k_{12}-k_{1}-k_{2}}^{k_{1}, k_{2}}\left(x_{1}, x_{2} ; h_{1}, h_{2}\right) S_{k-k_{12}-k_{3}}^{k_{12}, k_{3}}\left(x_{1}+x_{2}, x_{3} ; h_{1}+h_{2}, h_{3}\right) \\
& \quad=\sum_{k_{23}=k_{2}+k_{3}}^{k-k_{1}} h_{1}, h_{2}, h_{3} U_{k_{3}, k, k_{23}}^{k_{1}, k_{2}, k_{12}} S_{k-k_{1}-k_{23}}^{k_{1}, k_{23}}\left(x_{1}, x_{2}+x_{3} ; h_{1}, h_{2}+h_{3}\right) S_{k_{23}-k_{2}-k_{3}}^{k_{2}, k_{3}}\left(x_{2}, x_{3} ; h_{2}, h_{3}\right) .
\end{aligned}
$$

In [4] it was deduced that, when choosing $X=b^{+}+b^{-}$, the expression for the polynomial $S$ is given by

$$
S_{j}^{\left(k_{1}, k_{2}\right)}\left(x_{1}, x_{2} ; h_{1}, h_{2}\right)=\frac{1}{\sqrt{2^{j} j !}} H_{j}\left(\sqrt{\frac{h_{2}}{2 h_{1}\left(h_{1}+h_{2}\right)}} x_{1}-\sqrt{\frac{h_{1}}{2 h_{2}\left(h_{1}+h_{2}\right)}} x_{2}\right) .
$$

Using the expressions (3.17) and (2.12) in (3.16) yields (after some simple renamings) the following identity for Hermite polynomials [4, Formula 6.16]:

$H_{j}\left(a v_{1}+b v_{2}\right) H_{n}\left(-b v_{1}+a v_{2}\right)=\sum_{k=0}^{j+n}\left(\begin{array}{c}j+n \\ k\end{array}\right) a^{n+k} b_{2}^{j-k} F_{1}\left(\begin{array}{l}-n,-k \\ -j-n\end{array} ; \frac{1}{a^{2}}\right) H_{k}\left(v_{1}\right) H_{j+n-k}\left(v_{2}\right)$,

with $a^{2}+b^{2}=1$.

Remark 1 Note that in (3.18) the variables of the Hermite polynomials on the left side are an orthogonal transformation of the variables on the right side.

Remark 2 Recently Wünsche [16, 17], inspired by the following (alternative) definition of the Hermite polynomials:

$$
H_{m}(x)=\exp \left(-\frac{1}{4} \frac{\partial^{2}}{\partial x^{2}}\right)(2 x)^{m}
$$


introduced his so-called Hermite 2D polynomials

$$
H_{m, n}(A ; x, y)=\exp \left(-\frac{1}{4}\left(\frac{\partial^{2}}{\partial x^{2}}+\frac{\partial^{2}}{\partial y^{2}}\right)\right)\left(2 x^{\prime}\right)^{m}\left(2 y^{\prime}\right)^{n}
$$

where

$$
\left(\begin{array}{l}
x^{\prime} \\
y^{\prime}
\end{array}\right)=A\left(\begin{array}{l}
x \\
y
\end{array}\right)
$$

Herein, $A$ is an arbitrary two-dimensional matrix. Wünsche gave three different representations for the Hermite 2D polynomials. From one of them [16, Formula 2.11], it can be seen that the left hand side of (3.18) coincides with a Hermite 2D polynomial, provided $A$ is an orthogonal matrix. In this light it turns out that (3.18) is a special case of the representation [16, Formula 2.8] for Hermite 2D polynomials.

It is interesting to investigate the Granovskil and Zhedanov method for another form for the operator $X$. Let $X=H+N+b^{+}+b^{-}$(more general choices lead essentially to the same result); in this case one identifies (by considering its three-term recurrence) the polynomial $Q$ as being a Charlier polynomial [12]:

$$
\begin{aligned}
Q_{n}(x ; h, k) & =(-1)^{n} \sqrt{\frac{h^{n}}{n !}} C_{n}(x-k ; h) \\
& =(-1)^{n} \sqrt{\frac{h^{n}}{n !}}{ }_{2} F_{0}\left(\begin{array}{c}
-n,-x+k \\
-
\end{array}-\frac{1}{h}\right) .
\end{aligned}
$$

Using the definition of the $S$-polynomial

$$
S_{j}^{k_{1}, k_{2}}\left(x_{1}, x_{2} ; h_{1}, h_{2}\right)=\sum_{n_{1}+n_{2}=j} C_{n_{1}, n_{2}, 0}^{h_{1}, h_{2}, j} Q_{n_{1}}\left(x_{1} ; h_{1}, k_{1}\right) Q_{n_{2}}\left(x_{2} ; h_{2}, k_{2}\right),
$$

and the easily verified identity (expand the two hypergeometric series on the left side, interchange the order of summation and apply the binomial theorem)

$$
\sum_{n_{1}+n_{2}=j} \frac{(-1)^{n_{2}}}{n_{1} ! n_{2} !}{ }_{2} F_{0}\left(\begin{array}{c}
-n_{1}, y_{1} \\
-
\end{array} ; a_{1}\right){ }_{2} F_{0}\left(\begin{array}{c}
-n_{2}, y_{2} \\
-
\end{array} ; a_{2}\right)=\left(a_{2}\right)^{j} \frac{\left(y_{2}\right)_{j}}{j !}{ }_{2} F_{1}\left(\begin{array}{c}
-j, y_{1} \\
1-y_{2}-j
\end{array} ;-\frac{a_{1}}{a_{2}}\right)
$$

we arrive at the following explicit expression for $S$ (which we, for distinction with the 
Hermite-case, denote by $\mathcal{S})$ :

$$
\begin{aligned}
& \mathcal{S}_{j}^{k_{1}, k_{2}}\left(x_{1}, x_{2} ; h_{1}, h_{2}\right)=\sqrt{\frac{h_{1}^{j}}{j !\left(h_{1}+h_{2}\right)^{j} h_{2}^{j}}}\left(k_{2}-x_{2}\right)_{j}{ }_{2} F_{1}\left(\begin{array}{c}
-j, k_{1}-x_{1} \\
1-j-k_{2}+x_{2}
\end{array} ;-\frac{h_{2}}{h_{1}}\right) \\
& =\sqrt{\frac{h_{1}^{j}}{j !\left(h_{1}+h_{2}\right)^{j} h_{2}^{j}}}\left(k_{1}+k_{2}-x_{1}-x_{2}\right)_{j}{ }_{2} F_{1}\left(\begin{array}{c}
-j, k_{1}-x_{1} \\
k_{1}+k_{2}-x_{1}-x_{2}
\end{array} ; 1+\frac{h_{2}}{h_{1}}\right) .
\end{aligned}
$$

It is thus clear that in this case the $\mathcal{S}$-polynomial can be written in terms of a Meixner polynomial [12]:

$$
\begin{aligned}
\mathcal{S}_{j}^{k_{1}, k_{2}}\left(x_{1}, x_{2} ; h_{1}, h_{2}\right) & =\sqrt{\frac{h_{1}^{j}}{j !\left(h_{1}+h_{2}\right)^{j} h_{2}^{j}}}\left(k_{2}-x_{2}\right)_{j} M_{j}\left(x_{1}-k_{1} ; 1-j-k_{2}+x_{2}, \frac{h_{1}}{h_{1}+h_{2}}\right) \\
& =\sqrt{\frac{h_{1}^{j}}{j !\left(h_{1}+h_{2}\right)^{j} h_{2}^{j}}}\left(k_{1}+k_{2}-x_{1}-x_{2}\right)_{j} M_{j}\left(x_{1}-k_{1} ; k_{1}+k_{2}-x_{1}-x_{2},-\frac{h_{1}}{h_{2}}\right) .
\end{aligned}
$$

If we now plug all the basic ingredients into (3.16), we get a convolution identity for Meixner polynomials.

Theorem 3 The Meixner polynomials satisfy the following identity:

$$
\begin{aligned}
(1- & \left.j+x_{2}\right)_{j}(-s+j)_{n} M_{j}\left(x_{1} ; 1-j+x_{2}, \frac{b}{a} \frac{a-1}{b-1}\right) M_{n}\left(x_{1}+x_{2}-j ;-s+j, a\right) \\
= & \left(\frac{b}{a}\right)^{j+n}\left(\frac{a}{a-1}\right)^{j} \sum_{k=0}^{j+n}\left(\begin{array}{c}
j+n \\
k
\end{array}\right)\left(\frac{b-1}{b}\right)^{k}(-s+k)_{j+n-k}\left(1-k+x_{2}\right)_{k} \\
& { }_{2} F_{1}\left(\begin{array}{c}
-j,-j-n+k \\
-j-n
\end{array} ; \frac{a}{b}\right) M_{j+n-k}\left(x_{1} ;-s+k, b\right) M_{k}\left(s-x_{1}-x_{2} ; 1-k+x_{2}, \frac{b-1}{a-1}\right),
\end{aligned}
$$

where $s$ is some (arbitrary) constant.

Remark 4 We have written down two expressions in which the $\mathcal{S}$-polynomials are Meixner polynomials of degree $j$. There are four more of these expressions. This is because of the 24 Kummer solutions to the Gauss differential equation, there are six of them that have the first numerator parameter (in this case $-j$ ) fixed, see [11]. 
Remark 5 From the introductory section it is clear that for the multivariable case, we restrict ourselves to the Hermite case. One may ask oneself why this is the case. The reason is: orthogonality. Indeed if one replaces $x_{2}$ by $1-x_{1}$ in (3.17) it is immediately clear (and see also next section) that $S$ satisfies an orthogonality relation.

For the $\mathcal{S}$-case things are different. The Meixner polynomials also satisfy a (discrete) orthogonality, namely

$$
\sum_{x=0}^{\infty} \frac{(\beta)_{x}}{x !} c^{x} M_{m}(x ; \beta, c) M_{n}(x ; \beta, c)=\frac{c^{-n} n !}{(\beta)_{n}(1-c)^{\beta}} \delta_{m, n}, \beta>0 \text { and } 0<c<1 .
$$

We note that the conditions imposed on $\beta$ and $c$ are to ensure that the weight function is positive. However, the orthogonality is valid whenever $0<|c|<1$ and $\beta$ is not a negative integer. We cannot apply this orthogonality: e.g. in the case (3.27a) the parameter $\beta$ (after replacing $x_{2}$ by $1-x_{1}$ ) depends on $x_{1}$ which is clearly not allowed; in the case $(3.27 \mathrm{~b})$ the parameter $c$ does in general (i.e. for arbitrary values of $h_{1}$ and $h_{2}$ ) not meet its requirement $(0<|c|<1)$. The other four ways of writing $\mathcal{S}$ have similar problems.

\section{Multivariable Hermite polynomials}

When working with multivariable Hermite polynomials the classical region of integration is the real $n$-dimensional space $\mathbb{R}^{n}$. In [18, Section 2.3.4] the weight function $\exp \left(-\left(x_{1}^{2}+\cdots+x_{n}^{2}\right)\right)$ is considered and two different orthogonal bases are given. One of them is just a product of $n$ classical Hermite polynomials. In [19, Section 12.8] another generalization of Hermite polynomials is considered; these are orthogonal with respect to the weight function $\exp (-\varphi(x))$ with $\varphi(x)=\sum_{i, j} c_{i j} x_{i} x_{j}$ where $C$ is a symmetric, square, and positive definite matrix. The multivariable Hermite polynomials appearing in the $(n+1)$-fold tensor product are orthogonal over $\mathbb{R}^{n}$ for the weight function

$$
\exp (-\psi(x)), \text { with } \psi(x)=\left(x_{1}, x_{2}, \ldots, x_{n}, s-\sigma(x)\right) A\left(x_{1}, x_{2}, \ldots, x_{n}, s-\sigma(x)\right)^{t}
$$

where $A \in \mathbb{R}^{(n+1) \times(n+1)}, \sigma(x)=x_{1}+\cdots+x_{n}$ and $s$ some arbitrary constant. Moreover, it will turn out that

$$
A_{i j}=\frac{1}{2 h_{i}} \delta_{i j}-\frac{1}{2|h|}
$$


where $|h|=h_{1}+\cdots+h_{n+1}$.

We recall the expression for the two-variable $S$-polynomial, (where we have dropped the superscripts $k$ because $S$ is independent of them)

$$
S_{j}\left(x_{1}, x_{2} ; h_{1}, h_{2}\right)=\frac{1}{\sqrt{2^{j} j !}} H_{j}\left(\sqrt{\frac{h_{2}}{2 h_{1}\left(h_{1}+h_{2}\right)}} x_{1}-\sqrt{\frac{h_{1}}{2 h_{2}\left(h_{1}+h_{2}\right)}} x_{2}\right) .
$$

Since the Hermite polynomials are even resp. odd if their degree is even resp. odd, the $S$-polynomials have the following property:

$$
S_{j}\left(x_{1}, x_{2} ; h_{1}, h_{2}\right)=(-1)^{j} S_{j}\left(x_{2}, x_{1} ; h_{2}, h_{1}\right)
$$

This property has an interpretation in terms of the twist operation on binary coupling trees [8], see Figure 1, which in turn stems from the corresponding property of ClebschGordan coefficients of $\mathfrak{b}(1)$

$$
C_{n_{1}, n_{2}, n}^{h_{1}, h_{2}, j}=(-1)^{j} C_{n_{2}, n_{1}, n}^{h_{2}, h_{1}, j}
$$

(Note that this last equality is just Pfaff's transformation [20] for terminating ${ }_{2} F_{1}$-series.)

The second identity satisfied by the $S$-polynomials can be interpreted in terms of the rotation operation on binary coupling trees [8], see Figure 2. It is thus related to the two ways in which one can couple three $\mathfrak{b}(1)$ representations. We repeat it here:

$$
\begin{aligned}
& S_{k_{12}-k_{1}-k_{2}}\left(x_{1}, x_{2} ; h_{1}, h_{2}\right) S_{k-k_{12}-k_{3}}\left(x_{1}+x_{2}, x_{3} ; h_{1}+h_{2}, h_{3}\right) \\
& \quad=\sum_{k_{23}=k_{2}+k_{3}}^{k-k_{1}} h_{1}, h_{2}, h_{3} U_{k_{3}, k, k_{23}}^{k_{1}, k_{2}, k_{12}} S_{k-k_{1}-k_{23}}\left(x_{1}, x_{2}+x_{3} ; h_{1}, h_{2}+h_{3}\right) S_{k_{23}-k_{2}-k_{3}}\left(x_{2}, x_{3} ; h_{2}, h_{3}\right) .
\end{aligned}
$$

Notice how the left side of (4.34) follows from the tree on the left side of Figure 2. With each non-leaf node (i.e. with each intermediate or final coupling) one associates an $S$ polynomial. The first (resp. second) variable of this $S$-polynomial is the sum of all the variables associated with the leaves in the left (resp. right) subtree of the considered node. The same applies to the parameters $h_{i}$. The (positive integer) lower parameter is 
the difference between the value of the coupled representation $k$-label and the consisting $k$-labels. The $S$-polynomials on the right side of (4.34) are formed in the same way but working with the tree on the right side of the figure. The recoupling coefficient appearing in (4.34) is that associated with a recoupling of three representations as shown in Figure 2.

Now, we generalize the way the left hand side of (4.34) is formed and we associate with the tensor product of $n+1$ representations a product of $n S$-polynomials, each $S$-polynomial being associated with a non-leaf node of the binary coupling tree. From the decomposition (2.6) it follows that with each non-leaf node we can associate a non-negative integer. We will denote these by $l_{i}$, such that we write $R_{l}^{(h)}(x)$ for the resulting polynomial. In principle the notation $R_{l}^{(h)}(x)$ should also contain a reference to its defining binary coupling tree; for the moment however, we are dealing only with arbitrary but fixed trees, so we drop this reference in the notation.

Example 6 Consider the following tensor product of five $b(1)$ representations:

$$
\left(\left(\left(h_{1}, k_{1}\right) \otimes\left(h_{2}, k_{2}\right)\right) \otimes\left(h_{3}, k_{3}\right)\right) \otimes\left(\left(h_{4}, k_{4}\right) \otimes\left(h_{5}, k_{5}\right)\right)
$$

depicted by the binary coupling tree in Figure 3 (where we omitted the additive $h$-labels).

With this tensor product, we associate the following polynomial:

$$
\begin{aligned}
& R_{l_{1}, l_{2}, l_{3}, l_{4}}^{h_{1}, h_{2}, h_{3}, h_{4}, h_{5}}\left(x_{1}, x_{2}, x_{3}, x_{4}, x_{5}\right) \\
& =S_{l_{1}}\left(x_{1}, x_{2} ; h_{1}, h_{2}\right) S_{l_{2}}\left(x_{1}+x_{2}, x_{3} ; h_{1}+h_{2}, h_{3}\right) S_{l_{3}}\left(x_{4}, x_{5} ; h_{4}, h_{5}\right) \\
& \text { - } S_{l_{4}}\left(x_{1}+x_{2}+x_{3}, x_{4}+x_{5} ; h_{1}+h_{2}+h_{3}, h_{4}+h_{5}\right) \\
& =\frac{1}{\sqrt{2^{|l|} l_{1} ! l_{2} ! l_{3} ! l_{4} !}} H_{l_{1}}\left(\sqrt{\frac{h_{2}}{2 h_{1}\left(h_{1}+h_{2}\right)}} x_{1}-\sqrt{\frac{h_{1}}{2 h_{2}\left(h_{1}+h_{2}\right)}} x_{2}\right) \\
& \cdot H_{l_{2}}\left(\sqrt{\frac{h_{3}}{2\left(h_{1}+h_{2}\right)\left(h_{1}+h_{2}+h_{3}\right)}}\left(x_{1}+x_{2}\right)-\sqrt{\frac{h_{1}+h_{2}}{2 h_{3}\left(h_{1}+h_{2}+h_{3}\right)}} x_{3}\right) \\
& \cdot H_{l_{3}}\left(\sqrt{\frac{h_{5}}{2 h_{4}\left(h_{4}+h_{5}\right)}} x_{4}-\sqrt{\frac{h_{4}}{2 h_{5}\left(h_{4}+h_{5}\right)}} x_{5}\right) \\
& \cdot H_{l_{4}}\left(\sqrt{\frac{h_{4}+h_{5}}{2\left(h_{1}+h_{2}+h_{3}\right)(|h|)}}\left(x_{1}+x_{2}+x_{3}\right)-\sqrt{\frac{h_{1}+h_{2}+h_{3}}{2\left(h_{4}+h_{5}\right)(|h|)}}\left(x_{4}+x_{5}\right)\right) \text {. }
\end{aligned}
$$




\section{Change of variables}

We introduce $n$ new variables $v_{i}$, each $v_{i}$ being the argument of one of the Hermite polynomials in $R_{l}^{(h)}(x)$. This however does not lead to a unique correspondence between the $n+1$ variables $x$ and the $n$ variables $v$. To this end we introduce a linear constraint between the variables $x: x_{1}+\cdots+x_{n}+x_{n+1}=s$ with $s$ some arbitrary constant. Note that this is consistent with the definition of $\psi$ in (4.30).

The order in which the variables $v_{i}$ (and integers $l_{i}$ ) are associated to the non-leaf nodes is immaterial, but for the sake of explicitness we do this in post-order [9].

Example 7 For the tree in Figure 3 the connection between the variables $v$ and $x$ is the following:

$$
\left\{\begin{array}{l}
v_{1}=c_{1} x_{1}-d_{1} x_{2} \\
v_{2}=c_{2}\left(x_{1}+x_{2}\right)-d_{2} x_{3} \\
v_{3}=c_{3} x_{4}-d_{3} x_{5} \\
v_{4}=c_{4}\left(x_{1}+x_{2}+x_{3}\right)-d_{4}\left(x_{4}+x_{5}\right) \\
s=x_{1}+x_{2}+x_{3}+x_{4}+x_{5} .
\end{array}\right.
$$

Here, we have used abbreviations $c_{i}$ and $d_{i}$ for the rather tedious combinations of $h_{i}$ in (4.35). E.g.:

$$
c_{2}=\sqrt{\frac{h_{3}}{2\left(h_{1}+h_{2}\right)\left(h_{1}+h_{2}+h_{3}\right)}} \quad \text { and } \quad d_{2}=\sqrt{\frac{h_{1}+h_{2}}{2 h_{3}\left(h_{1}+h_{2}+h_{3}\right)}} .
$$

The equations (4.36) can also be written in matrix form (setting $s=v_{5}$ ):

$$
\left(\begin{array}{ccccc}
c_{1} & -d_{1} & 0 & 0 & 0 \\
c_{2} & c_{2} & -d_{2} & 0 & 0 \\
0 & 0 & 0 & c_{3} & -d_{3} \\
c_{4} & c_{4} & c_{4} & -d_{4} & -d_{4} \\
1 & 1 & 1 & 1 & 1
\end{array}\right)\left(\begin{array}{l}
x_{1} \\
x_{2} \\
x_{3} \\
x_{4} \\
x_{5}
\end{array}\right)=\left(\begin{array}{l}
v_{1} \\
v_{2} \\
v_{3} \\
v_{4} \\
v_{5}
\end{array}\right) .
$$

Since the arguments of the Hermite polynomials are linear combinations of the variables $x$, one can write (because $T$ is non-singular, see (4.56))

$$
T \cdot X=V \Longleftrightarrow X=T^{-1} \cdot V
$$


where $X$ and $V$ are both column vectors, and $T \in \mathbb{R}^{(n+1) \times(n+1)}$. From (4.39) it is clear that the $k$-th row of the matrix $T$ concerns the variable $v_{k}$, while the $i$-th column concerns the variable $x_{i}$. Using the notation $c_{k}$ and $d_{k}$ we have

$$
T_{k i}=\left\{\begin{array}{ll}
c_{k} & \text { if } x_{i} \text { is in the left subtree of } v_{k} \\
-d_{k} & \text { if } x_{i} \text { is in the right subtree of } v_{k} \\
0 & \text { otherwise }
\end{array} \quad \text { for } 1 \leq k \leq n \text { and } 1 \leq i \leq n+1,\right.
$$

and

$$
T_{n+1, i}=1 \quad \text { for } 1 \leq i \leq n+1 \text {. }
$$

The last row of $T$ corresponds of course to the constraint $x_{1}+\cdots+x_{n+1}=s=v_{n+1}$.

Making the notation $c_{k}$ and $d_{k}$ explicit we have:

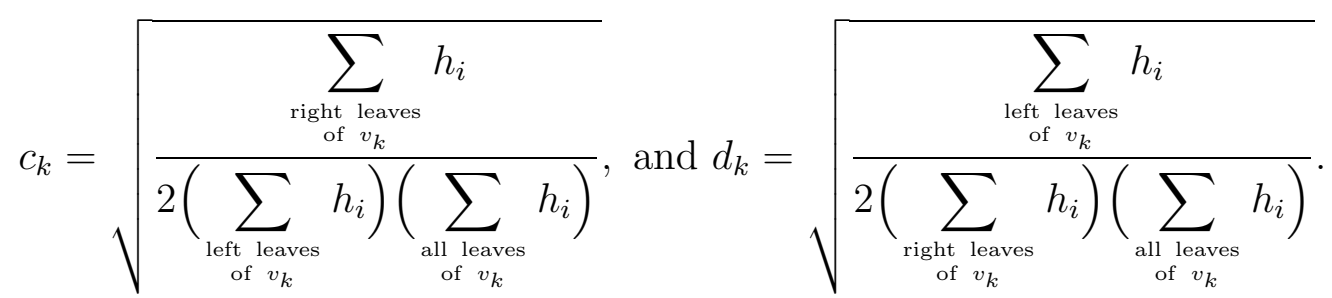

When the $R$-polynomial is transformed using (4.39) it becomes, by definition of the transformation, a product of $n$ independent Hermite polynomials. The orthogonality for the Hermite polynomials will be applicable provided that $\psi(x)$ of (4.30) transforms into $v_{1}^{2}+\cdots+v_{n}^{2}$, i.e.:

$$
\begin{aligned}
\left(x_{1}, \ldots, x_{n+1}\right) A\left(x_{1}, \ldots, x_{n+1}\right)^{t} & =\left(v_{1}, \ldots, v_{n+1}\right)\left(T^{-1}\right)^{t} A T^{-1}\left(v_{1}, \ldots, v_{n+1}\right)^{t} \\
& =v_{1}^{2}+\cdots+v_{n}^{2} .
\end{aligned}
$$

This, in turn, is equivalent to

$$
\left(T^{-1}\right)^{t} A T^{-1}=\left(\begin{array}{cc}
I_{n} & \mathcal{O}_{n \times 1} \\
\mathcal{O}_{1 \times n} & 0
\end{array}\right) \Longleftrightarrow A=T^{t}\left(\begin{array}{cc}
I_{n} & \mathcal{O}_{n \times 1} \\
\mathcal{O}_{1 \times n} & 0
\end{array}\right) T
$$


where $I_{n}$ denotes the identity matrix of order $n$ and $\mathcal{O}_{n \times m}$ is the zero-matrix of the given order. This last equality provides us a way for calculating the explicit form of the matrix $A$, namely

$$
A_{i j}=\sum_{k=1}^{n} T_{k i} T_{k j} \text { for } 1 \leq i, j \leq n+1
$$

Lemma 8 For an arbitrary binary coupling tree on $n+1$ leaves with leaf labels $\left(h_{i}, k_{i}\right)$, $i=1, \ldots n+1$, and with transformation matrix $T$ defined by (4.40), we have

$$
A_{i j}=\sum_{k=1}^{n} T_{k i} T_{k j}=\frac{1}{2 h_{i}} \delta_{i j}-\frac{1}{2|h|} .
$$

The matrix $A$ is thus independent of the shape of the binary coupling tree.

Proof: Let $n l_{n}$ (resp. $n r_{n}$ ) denote the number of leaves in the left (resp. right) subtree of $v_{n}$, the root node of the tree. We can then split up our formula into three parts:

$$
A_{i j}=\sum_{k=1}^{n} T_{k i} T_{k j}=\sum_{k=1}^{n l_{n}-1} T_{k i} T_{k j}+\sum_{k=n l_{n}}^{n-1} T_{k i} T_{k j}+T_{n i} T_{n j} .
$$

Since the first (resp. second) sum of (4.46) corresponds to the $A$-matrix of the left (resp. right) subtree of the root, we use induction on the number of internal nodes in the tree. It is easily verified that the desired result is true for small values of $n$.

There are (essentially) two cases to consider:

1. The variables $x_{i}$ and $x_{j}$ belong both to the same subtree of $v_{n}$,

2. the variables $x_{i}$ and $x_{j}$ belong to different subtrees of $v_{n}$.

Let $x_{i}$ and $x_{j}$ both belong to the same, say left, subtree of $v_{n}$. This implies $T_{n i}=T_{n j}=c_{n}$, and thus

$$
\begin{aligned}
\sum_{k=1}^{n} T_{k i} T_{k j} & =\left(\frac{1}{2 h_{i}} \delta_{i j}-\frac{1}{2\left(h_{1}+\cdots+h_{n l_{n}}\right)}\right)+0+\frac{\left(h_{n l_{n}+1}+\cdots+h_{n+1}\right)}{2|h|\left(h_{1}+\cdots+h_{n l_{n}}\right)} \\
& =\frac{1}{2 h_{i}} \delta_{i j}-\frac{1}{2|h|} .
\end{aligned}
$$


where the first sum in (4.46) is evaluated using the induction hypothesis, the second sum is zero because $T_{k i}=T_{k j}=0$ for $k=n l_{n}, \ldots, n-1$, and the third term is simply $c_{n}^{2}$. (We assumed, for the sake of simplicity, that the leaves in the left subtree have labels $h_{1}$ up to $\left.h_{n l_{n}} \cdot\right)$

Secondly, let $x_{i}$ belong to the left subtree and $x_{j}$ to the right subtree. In this case $T_{k j}=0$ for $k=1, \ldots, n l_{n}-1$ and $T_{k i}=0$ for $k=n l_{n}, \ldots, n-1$. This implies

$$
\sum_{k=1}^{n} T_{k i} T_{k j}=-c_{n} d_{n}=-\frac{1}{2|h|} .
$$

Thus we have established that the weight function (4.30) after transformation with (4.39) becomes a product of $n$ classical Hermite weights.

\section{The Jacobian of the transformation}

The transformation between the variables $x$ and $v$ is a simple linear transformation. If we want to show the orthogonality of the $R$-polynomials, we need the Jacobian of the transformation, i.e. we want to know

$$
J=\left|\operatorname{det} \frac{\partial x_{i}}{\partial v_{k}}\right|, \text { with } 1 \leq i, k \leq n
$$

From (4.39) it is immediately clear that

$$
\operatorname{det} \frac{\partial v_{k}}{\partial x_{i}}=\operatorname{det} T \text { for } 1 \leq i, k \leq n+1 \text {. }
$$

However, we need the Jacobian between the $n$ independent variables $x_{i}$ and the $n$ variables $v_{k}$. The linear constraint between the $n+1$ variables $x_{i}$ is equivalent to subtracting the last column of the matrix $T$ from all its other columns; this does not affect the value of the determinant of $T$. Since the $(n+1)$-th row of $T$ now entirely consists of zeros, except for the last element, we have

$$
\operatorname{det} \frac{\partial v_{k}}{\partial x_{i}}=\operatorname{det} T \text { for } 1 \leq i, k \leq n \text {. }
$$


Since we use a linear transformation we now have

$$
J=\left|\operatorname{det} \frac{\partial x_{i}}{\partial v_{k}}\right|=\left|\frac{1}{\operatorname{det} T}\right|
$$

It is thus sufficient to calculate the determinant of the matrix $T$.

Example 9 For the running example, the transformation matrix $T$ equals:

$$
T=\left(\begin{array}{ccccc}
c_{1}^{\star} & -d_{1}^{\star} & 0^{\star} & 0 & 0 \\
c_{2}^{\star} & c_{2}^{\star} & -d_{2}^{\star} & 0 & 0 \\
0 & 0 & 0 & c_{3}^{\ddagger} & -d_{3}^{\ddagger} \\
c_{4} & c_{4} & c_{4} & -d_{4} & -d_{4} \\
1^{\star} & 1^{\star} & 1^{\star} & 1^{\ddagger} & 1^{\ddagger}
\end{array}\right) .
$$

We want to determine $|\operatorname{det} T|$ and to this end we transform $T$ into an upper triangular matrix, using column transformations only.

In (4.53), the nine elements annotated with a $\star$ constitute the $3 \times 3$ transformation matrix associated with the left subtree, and analogously for the four elements annotated with a ‡. Perform the column transformations, $C_{1} \leftarrow C_{1}-C_{2}, C_{2} \leftarrow C_{2}-C_{3}$, and $C_{4} \leftarrow C_{4}-C_{5}$ on $T$ and one gets:

$$
T=\left(\begin{array}{ccccc}
c_{1}+d_{1}^{\star} & -d_{1}^{\star} & 0^{\star} & 0 & 0 \\
0^{\star} & c_{2}+d_{2}^{\star} & -d_{2}^{\star} & 0 & 0 \\
0 & 0 & 0 & c_{3}+d_{3}^{\ddagger} & -d_{3}^{\ddagger} \\
0 & 0 & c_{4} & 0 & -d_{4} \\
0^{\star} & 0^{\star} & 1^{\star} & 0^{\ddagger} & 1^{\ddagger}
\end{array}\right) .
$$

Note that the nine starred elements form an upper triangular matrix; the same applies to the four double-daggered elements. Moreover the column transformations performed so far only concerned either the left or the right subtree, but not both.

Subtracting the fifth column from the third and swapping columns yields an upper triangular matrix, with determinant:

$$
\left(c_{1}+d_{1}\right)\left(c_{2}+d_{2}\right)\left(c_{3}+d_{3}\right)\left(c_{4}+d_{4}\right) \text {. }
$$


We can easily extend the method of this example to prove the following lemma.

Lemma 10 For an arbitrary binary coupling tree on $n+1$ leaves with labels $\left(h_{i}, k_{i}\right)$, $i=1, \ldots, n+1$ and with transformation matrix $T$ determined by (4.40), we have

$$
|\operatorname{det} T|=\prod_{i=1}^{n}\left(c_{i}+d_{i}\right)=\sqrt{\frac{|h|}{2^{n}\left(h_{1} \cdot h_{2} \cdots h_{n+1}\right)}} .
$$

Proof: Once again we let $n l_{n}$ (resp. $n r_{n}$ ) denote the number of leaves in the left (resp. right) subtree of $v_{n}$. In general the matrix $T$ has the following structure:

$$
T=\left(\begin{array}{cc}
\tilde{T}_{l} & \mathcal{O}_{\left(n l_{n}-1\right) \times n r_{n}} \\
\mathcal{O}_{\left(n r_{n}-1\right) \times n l_{n}} & \tilde{T}_{r} \\
c_{n}, \ldots, c_{n} & -d_{n}, \ldots,-d_{n} \\
1, \ldots, 1 & 1, \ldots, 1
\end{array}\right)
$$

Herein, $\tilde{T}_{l}$ (resp. $\tilde{T}_{r}$ ) is a part of the matrix associated with the left (resp. right) subtree of $v_{n}$. More particularly,

$$
\left(\begin{array}{c}
\tilde{T}_{l} \\
1, \ldots, 1
\end{array}\right)
$$

is the matrix associated with the left subtree of $v_{n}$.

The structure of the matrix suggests to use induction on the order of the matrix. We thus assume that we can transform matrices smaller than $T$ into upper triangular matrices, using column transformations only, and that the elements on the diagonal are of the form $c_{i}+d_{i}$ (and one element 1). Schematically:

$$
\left(\begin{array}{c}
\tilde{T}_{l} \\
1, \ldots, 1
\end{array}\right) \stackrel{\text { column tf }}{\longrightarrow}\left(\begin{array}{ccccc}
c_{1}+d_{1} & & & & \\
0 & c_{2}+d_{2} & & & \\
0 & 0 & \ddots & & \\
0 & \ldots & 0 & c_{n l_{n}-1}+d_{n l_{n}-1} & \\
0 & \ldots & 0 & 0 & 1
\end{array}\right)
$$

Performing the same column transformations on the extended matrix yields:

$$
\left(\begin{array}{c}
\tilde{T}_{l} \\
\mathcal{O}_{\left(n r_{n}-1\right) \times n l_{n}} \\
c_{n}, \ldots, c_{n} \\
1, \ldots, 1
\end{array}\right) \stackrel{\text { column tf }}{\longrightarrow}\left(\begin{array}{ccccc} 
& T_{l}^{\prime} & & * \\
& & \mathcal{O}_{\left(n r_{n}-1\right) \times n l_{n}} & & \\
0 & \ldots & 0 & 0 & c_{n} \\
0 & \ldots & 0 & 0 & 1
\end{array}\right)
$$


where $\tilde{T}_{l}^{\prime}$ is a square upper triangular matrix of the order $n l_{n}-1$ with diagonal elements $c_{i}+d_{i}$, where $i=1, \ldots, n l_{n}-1$. The $*$ stands for some column vector.

One can apply the same arguments to the matrix corresponding to the right subtree of $v_{n}$, finally leading to

$$
T=\left(\begin{array}{cc}
\tilde{T}_{l} & \mathcal{O}_{\left(n l_{n}-1\right) \times n r_{n}} \\
\mathcal{O}_{\left(n r_{n}-1\right) \times n l_{n}} & \tilde{T}_{r} \\
c_{n}, \ldots, c_{n} & -d_{n}, \ldots,-d_{n} \\
1, \ldots, 1 & 1, \ldots, 1
\end{array}\right) \rightarrow\left(\begin{array}{cccc}
\tilde{T}_{l}^{\prime} & * & \mathcal{O}_{\left(n l_{n}-1\right) \times n r_{n}} & \\
\mathcal{O}_{\left(n r_{n}-1\right) \times n l_{n}} & & \tilde{T}_{r}^{\prime} & * \\
0, \ldots, 0 & c_{n} & 0, \ldots, 0 & -d_{n} \\
0, \ldots, 0 & 1 & 0, \ldots, 0 & 1
\end{array}\right)
$$

Subtracting the last column from the $n l_{n}$-th column and switching columns, transforms $T$ into an upper triangular matrix with diagonal elements $c_{i}+d_{i}$, with $i=1, \ldots n$ and one diagonal element that equals one. The (absolute value of the) determinant of $T$ is thus

$$
|\operatorname{det}(T)|=\left(c_{1}+d_{1}\right) \cdot\left(c_{2}+d_{2}\right) \cdots\left(c_{n}+d_{n}\right) .
$$

Rewriting this in terms of parameters $h_{i}$ is easy using induction and the simple fact that

$$
c_{n}+d_{n}=\sqrt{\frac{|h|}{2\left(h_{1}+\cdots+h_{n l_{n}}\right)\left(h_{n l_{n}+1}+\cdots+h_{n+1}\right)}} .
$$

This completes the proof of (4.56), and thus

$$
J=\sqrt{\frac{2^{n}\left(h_{1} \cdot h_{2} \cdots h_{n+1}\right)}{|h|}} .
$$

\section{The orthogonality explicitly}

It is immediately clear that if $x$ and $v$ are connected through (4.39), that $x \in \mathbb{R}^{n} \Longleftrightarrow$ $v \in \mathbb{R}^{n}$.

With this last simple observation we are now in the position to prove the orthogonality of the $R$-polynomials. 
Theorem 11 With the tensor product of $n+1 \mathfrak{b}(1)$ representations labeled $\left(h_{i}, k_{i}\right)$, with $i=1, \ldots, n+1$, i.e. with every binary coupling tree with $n$ internal nodes, we associate a set of polynomials $R_{l}^{(h)}(x)$ in $n$ variables. These polynomials are orthogonal on $\mathbb{R}^{n}$ for the weight function

$w^{(h)}(x)=\exp (-\psi(x))$, with $\psi(x)=\left(x_{1}, x_{2}, \ldots, x_{n}, s-\sigma(x)\right) A\left(x_{1}, x_{2}, \ldots, x_{n}, s-\sigma(x)\right)^{t}$,

and

$$
A_{i j}=\frac{1}{2 h_{i}} \delta_{i j}-\frac{1}{2|h|}
$$

Explicitly the orthogonality reads:

$$
\int_{\mathbb{R}^{n}} R_{l}^{(h)}(x) R_{l^{\prime}}^{(h)}(x) w^{(h)}(x) d x=\sqrt{\frac{(2 \pi)^{n} h_{1} \cdots \cdot h_{n+1}}{|h|}} \delta_{l, l^{\prime}} .
$$

Proof: The proof is easy since we have collected all the ingredients in this section. We have:

$$
\begin{aligned}
\int_{\mathbb{R}^{n}} R_{l}^{(h)}(x) R_{l^{\prime}}^{(h)}(x) w^{(h)}(x) d x & =\int_{\mathbb{R}^{n}} J \prod_{i=1}^{n} \frac{H_{l_{i}}\left(v_{i}\right)}{\sqrt{2^{l_{i}} l_{i} !}} \frac{H_{l_{i}^{\prime}}\left(v_{i}\right)}{\sqrt{2^{l^{\prime}} l_{i}^{\prime} !}} e^{-v_{i}^{2}} d v_{i} \\
& =J \sqrt{\pi^{n}} \delta_{l, l^{\prime}} \\
& =\sqrt{\frac{(2 \pi)^{n} h_{1} \cdots h_{n+1}}{|h|}} \delta_{l, l^{\prime}} .
\end{aligned}
$$

Remark 12 It is an easy calculation to verify that $\psi(x)$, when written explicitly in terms of $x_{i}(1 \leq i \leq n)$, has the following form:

$$
\psi(x)=\sum_{i=1}^{n} \frac{1}{2 h_{i}} x_{i}^{2}+\frac{1}{2 h_{n+1}}\left(\sum_{i=1}^{n} x_{i}\right)^{2}-\frac{s}{h_{n+1}} \sum_{i=1}^{n} x_{i}+s^{2}\left(\frac{1}{2 h_{n+1}}-\frac{1}{2|h|}\right) .
$$

From this it is easily seen that the quadratic part of $\psi(x)$ is positive definite, which is neccessary for the integrability of the left hand side of (4.65). Observe that the minimum of $\psi(x)$ (i.e. the maximum of $w^{(h)}(x)$ ) occurs at $x_{i}=s h_{i} /|h|, 1 \leq i \leq n$. 
Theorem 11 is correct for any value of $s$. For each value of $s$ one gets a different weight function and different polynomials. Thus, both the weight function and the polynomials $R_{l}^{(h)}(x)$ depend on $s$; in both cases the reference to $s$ is dropped to make the notation easier. When $s=0$ the weight function simplifies and $\psi$ becomes a purely quadratic form, which is thus as in [19].

\section{An integral formula for recoupling coefficients}

In this section we will show that the set of polynomials $R_{l, T}^{(h)}(x)$ associated with a fixed binary coupling tree $T$ and fixed representation labels $\left(h_{i}, k_{i}\right)$, but varying values $l_{i}$ forms a basis for $\Pi^{n}$. This means that a polynomial $R_{l, T_{1}}^{(h)}(x)$ (l fixed) associated with a binary coupling tree $T_{1}$ can be written as a linear combination of polynomials $R_{l^{\prime}, T_{2}}^{(h)}(x)$ ( $l^{\prime}$ variable) associated with another binary coupling tree $T_{2}$. The connection coefficients between these two bases are the recoupling coefficients of $\mathfrak{b}(1)$. The orthogonality of the $R$-polynomials, shown in Theorem 11, leads to an integral formula for these recoupling coefficients.

We start with a simple observation: for any binary coupling tree the degree of $R_{l}^{(h)}(x)$ equals $|l|$. This follows immediately from the fact that $S_{j}\left(x_{1}, x_{2} ; h_{1}, h_{2}\right)$ is a polynomial of degree $j$ in $x_{1}$ and $x_{2}$.

Theorem 13 For any binary coupling tree on $n+1$ leaves, with fixed representation labels $\left(h_{i}, k_{i}\right)(i=1, \ldots, n+1)$, the set of polynomials $R_{l}^{(h)}(x)$ associated with it forms a basis for $\Pi^{n}$.

Proof: The orthogonality of the polynomials $R_{l}^{(h)}(x)$ immediately implies their linear independence. The theorem then follows by the same counting argument as in $[5$, Theorem $20]$.

Since the $S$-polynomials satisfy the two basic properties (4.32) and (4.34), which are identical to the properties of the $S$-polynomials in the $\mathfrak{s u}(1,1)$-case (see [5, Formulas 2.11 
and 2.12]), one can use exactly the same reasoning as in [5, Theorem 21] to prove the following theorem.

Theorem 14 Consider a binary coupling tree $T_{1}$, with fixed values $\left(h_{j}, k_{j}\right)$ and $l_{i}$. Consider a second binary coupling tree $T_{2}$ with the same fixed values $\left(h_{j}, k_{j}\right)$ but varying values $l_{i}^{\prime}$, such that $|l|=\left|l^{\prime}\right|$. Then the polynomial $R_{l, T_{1}}^{(h)}(x)$ can be written as a linear combination of polynomials $R_{l^{\prime}, T_{2}}^{(h)}(x)$ :

$$
R_{l, T_{1}}^{(h)}(x)=\sum_{\left|l^{\prime}\right|=|l|} C_{l^{\prime}} R_{l^{\prime}, T_{2}}^{(h)}(x) .
$$

The connection coefficient $C_{l^{\prime}}$ is equal to the $3 n j$-coefficient $\left\langle T_{1}(l), T_{2}\left(l^{\prime}\right)\right\rangle$.

Corollary 15 For two arbitrary binary coupling trees $T_{1}$ and $T_{2}$ with the same representation labels $\left(h_{j}, k_{j}\right)$ the recoupling coefficient $\left\langle T_{1}(l), T_{2}\left(l^{\prime}\right)\right\rangle$ is given by:

$$
\left\langle T_{1}(l), T_{2}\left(l^{\prime}\right)\right\rangle=\sqrt{\frac{|h|}{(2 \pi)^{n} h_{1} \cdots \cdot h_{n+1}}} \int_{\mathbb{R}^{n}} R_{l, T_{1}}^{(h)}(x) R_{l^{\prime}, T_{2}}^{(h)}(x) w^{(h)}(x) d x,
$$

with $w^{(h)}(x)$ as in Theorem 11.

Proof: The result follows immediately from combining Theorems 11 and 14 .

Remark 16 In Section III we saw that the addition formula (3.18) is a direct consequence of the basic relation (3.16) for $S$-polynomials. Clearly, (5.67) is an extension of this relation. If one changes from variables $x$ to $v$ on the right side of (5.67), the variables on the left side will be an orthogonal transformation of the variables $v$. This can be seen be using the "method of trees" and repeated application of (3.16). It can also be understood by the fact that $\exp \left(-\left(v_{1}^{2}+\cdots+v_{n}^{2}\right)\right)$ is invariant under orthogonal transformations.

\section{References}

[1] N.J. Vilenkin and A.U. Klimyk, Representation of Lie Groups and Special Functions, Vol 1. (Kluwer Academic Publishers, 1991.) 
[2] J. Van der Jeugt, 3nj-Coefficients and orthogonal polynomials of hypergeometric type, in Lecture Notes in Mathematics, vol. 1817: Orthogonal Polynomials and Special Functions. Eds. Erik Koelink and Walter Van Assche (Springer, Berlin, 2003).

[3] Y.I. Granovskiŭ and A.S. Zhedanov, J. Phys. A, 26, 4339 (1993).

[4] J. Van der Jeugt, J. Math. Phys., 38, 2728 (1997).

[5] S. Lievens and J. Van der Jeugt, J. Math. Phys., 43, 3824 (2002).

[6] L. C. Biedenharn and J. D. Louck, The Racah-Wigner Algebra in Quantum Theory, Encyclopedia of Mathematics and its Applications, Vol 9 (Addison-Wesley, Reading, MA, 1981).

[7] V. Fack, S. N. Pitre, and J Van der Jeugt, Comput. Phys. Commun., 83, 275 (1994).

[8] V. Fack, S. Lievens, and J. Van der Jeugt, Comput. Phys. Commun., 119, 99 (1999).

[9] D. E. Knuth, Fundamental Algorithms, The Art of Computer Programming, Vol 1 (Addison-Wesley, Reading, MA, third edition, 1997).

[10] W. N. Bailey, Generalized Hypergeometric Series. (Cambridge University Press, $1935)$.

[11] L. J. Slater, Generalized Hypergeometric Functions. (Cambride University Press, 1966).

[12] R. Koekoek and R. F. Swarttouw. "The Askey-scheme of hypergeometric orthogonal polynomials and its $q$-analogue." Technical Report 98-17, Delft University of Technology, 1998.

[13] W. Jr. Miller. Lie Theory and Special Functions, Mathematics in Science and Engineering, Vol 43 (Academic Press, New York and London, 1968).

[14] A. Zhedanov, J. Phys. A, 30, 8337 (1997).

[15] H. T. Koelink and J. Van der Jeugt, SIAM J. Math. Anal., 29, 794 (1998). 
[16] A. Wünsche, J. Comput. Appl. Math., 133, 665 (2001).

[17] A. Wünsche, J. Phys. A, 33, 1603 (2000).

[18] C. F. Dunkl and Y. Xu, Orthogonal Polynomials of Several Variables, Encyclopedia of Mathematics and its Applications, Vol 81 (Cambridge University Press, 2001).

[19] A. Erdélyi, Higher Transcendental Functions, Vol 2. (Mc-Gray-Hill Book Company, 1953).

[20] G. E. Andrews, R. Askey, and R. Roy, Special Functions, Encyclopedia of Mathematics and its Applications, Vol 71 (Cambridge University Press, 1999). 


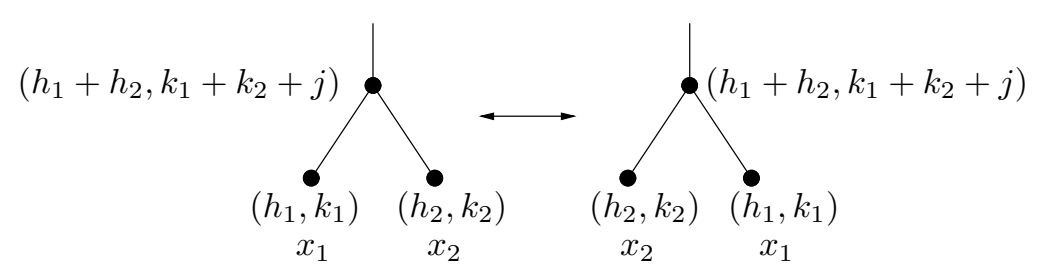

Figure 1: 


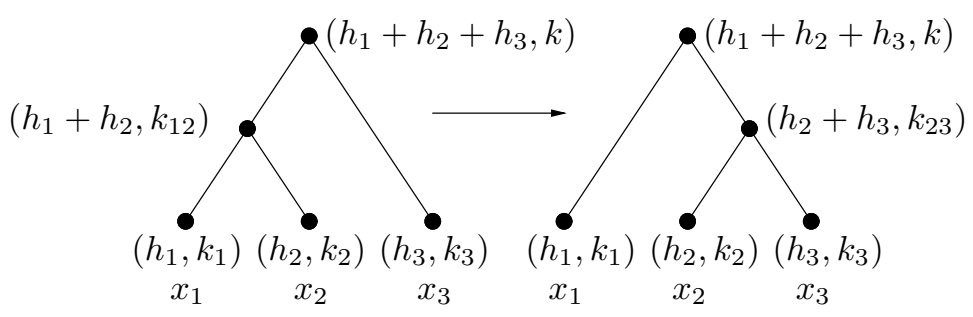

Figure 2: 


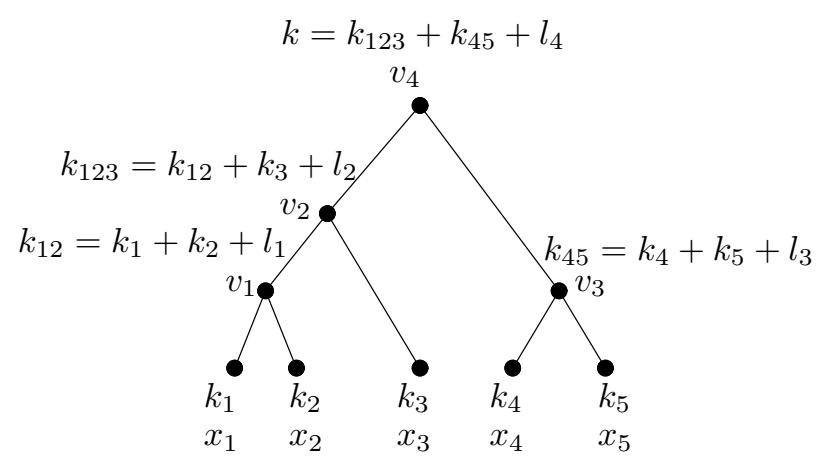

Figure 3: 


\section{Captions for Figures}

1. A twist operation on binary coupling trees

2. A rotation on binary coupling trees

3. A binary coupling tree corresponding to the tensor product of five $\mathfrak{b}(1)$ representations 